\title{
Influenza A (H6N6) virus isolated from chickens replicate in mice and human lungs without prior adaptation
}

\author{
Lingxi Gao ${ }^{1}$, Xijing Wang ${ }^{1}$, Yugui Lin ${ }^{1}$, Weijuan Zhong ${ }^{1}$, Shanggui Su${ }^{1}$, Siyu Zhou ${ }^{1}$, \\ Yinchuan Zhu ${ }^{1}$, Chengyi $\mathrm{Li}^{1}$, Xiaohui Fan ${ }^{1}$, and Zengfeng Zhang ${ }^{1}$ \\ ${ }^{1}$ Guangxi Medical University
}

July 4, 2021

\begin{abstract}
H6H6 subtype avian influenza virus (AIV) is currently prevalent in wild birds and poultry. Its host range has gradually expanded to the mammals, such as swine. Some strains of H6N6 AIV have even acquired the ability to bind to human-like receptors SA- $\alpha 2,6 \mathrm{Gal}$, thus increasing the risk of animal-human transmission. To investigate whether H6N6 AIV can cross interspecies barriers from poultry to mammals and even humans, we assessed the molecular characteristics, receptor-binding preference, replication in mice, and the human lung of three chicken-originated H6N6 strains. Among the three chicken-originated H6N6 strains, A/CK/ZZ/346/2014 (ZZ346) virus with P186T, H156R, S263G mutation of hemagglutinin (HA) showed the ability to bind to avian-like $\mathrm{SA} \alpha-2,3 \mathrm{Gal}$ and human-like $\mathrm{SA} \alpha-2,6 \mathrm{Gal}$ receptors. Moreover, H6N6 viruses, especially the ZZ346 strain, could replicate and infect mice and human lungs. Our study detected the H6N6 virus favorable to bind to both avian-like $\mathrm{SA} \alpha-2,3 \mathrm{Gal}$ and human-like SA $\alpha-2,6 \mathrm{Gal}$ receptors, verified its ability to cross the species barrier to infect mice, and verified to infect human lungs without prior adaptation. This study emphasizes the importance of continuous and intense monitoring of the evolution of the H6N6 virus in the terrestrial bird.
\end{abstract}

Influenza A (H6N6) virus isolated from chickens replicate in mice and human lungs without prior adaptation

Lingxi $\mathrm{Gao}^{1+}$,Xijing Wang ${ }^{1+}$, Yugui Lin $^{1+}$, Weijuan Zhong ${ }^{1}$, Shanggui Su${ }^{1}$, Siyu Zhou ${ }^{1}$, Yinchuan Zhu ${ }^{1}$, Chengyi Li $^{1}$,Xiaohui Fan $^{1}{ }^{*}$, Zengfeng Zhang ${ }^{1 *}$

${ }^{1}$ Department of Microbiology, Guangxi Medical University, Nanning 530021, Guangxi, China

+ These authors have contributed equally to this work.

\section{* Correspondence:}

Zengfeng Zhang

E-mail: zfzhangphd@163.com

Xiaohui Fan

E-mail: fanxiaohui63@163.com

Authers' information:

Keywords: influenza A virus, H6N6, mice, human, infection

\section{ABSTRACT}

H6H6 subtype avian influenza virus (AIV) is currently prevalent in wild birds and poultry. Its host range has gradually expanded to the mammals, such as swine. Some strains of H6N6 AIV have even acquired 
the ability to bind to human-like receptors SA- $\alpha 2,6 \mathrm{Gal}$, thus increasing the risk of animal-human transmission. To investigate whether H6N6 AIV can cross interspecies barriers from poultry to mammals and even humans, we assessed the molecular characteristics, receptor-binding preference, replication in mice, and the human lung of three chicken-originated H6N6 strains. Among the three chicken-originated H6N6 strains, A/CK/ZZ/346/2014 (ZZ346) virus with P186T, H156R, S263G mutation of hemagglutinin (HA) showed the ability to bind to avian-like SA $\alpha-2,3 \mathrm{Gal}$ and human-like $\mathrm{SA} \alpha-2,6 \mathrm{Gal}$ receptors. Moreover, H6N6 viruses, especially the ZZ346 strain, could replicate and infect mice and human lungs. Our study detected the H6N6 virus favorable to bind to both avian-like SA $\alpha-2,3 \mathrm{Gal}$ and human-like SA $\alpha-2,6 \mathrm{Gal}$ receptors, verified its ability to cross the species barrier to infect mice, and verified to infect human lungs without prior adaptation. This study emphasizes the importance of continuous and intense monitoring of the evolution of the H6N6 virus in the terrestrial bird.

\section{KEYWORDS}

avian influenza virus, H6N6 subtype, replicate, infect, mice, human

\section{INTRODUCTION}

Since December 2019, the global outbreak of coronavirus disease 2019 (COVID-19) has seriously threatened human health and public health security. We are currently making every effort to prevent and control the COVID-19; however, it is impossible to ignore the harm the influenza virus can inflict on humans (F. Wu et al., 2020). In the past two decades, highly pathogenic avian influenza viruses H5N1, H7N9, and H5N6 continuously crossed the species barrier from poultry to humans (Claas et al., 1998; Gao et al., 2013; Shen et al., 2016). Low-pathogenic H6 subtype influenza virus was also isolated from a patient diagnosed of lower respiratory tract infection in 2013, and a highly close H6 virus was subsequently isolated from a dog in 2014 (Wei et al., 2013), indicating H6 subtype virus could crossed the species barriers to mammals as well thus posing a potential threat to human health.

H6 subtype influenza A virus was first isolated from turkey in 1963 (Lupiani \& Reddy, 2009). Since then, H6 subtype AIV has widely spread around the world. It is mainly divided into North American and Eurasian lineages, where the Eurasian lineage can be further divided into Group I (ST339-like), Group II (ST2853like), Group III (HN573-like) (Bahl, Vijaykrishna, Holmes, Smith, \& Guan, 2009; Wallensten et al., 2005; zu Dohna, Li, Cardona, Miller, \& Carpenter, 2009), and W312-like minor poultry group (Chin et al., 2002). In China's live poultry market, H6 viruses (including H6N1, H6N2, H6N5, H6N6, H6N8, etc.) have been transmitted to poultry and prevalent for a long time (Huang et al., 2012; Zhao et al., 2011). From 2000 to 2005, ST339-like Group I H6N2 was the most frequently detected H6 subtype in live poultry markets in southern China. However, since the emergence of the H6N6 virus in 2005, the ST2853-like Group II H6N6 has gradually replaced the H6N2 subtype. Similar group replacement scenario has also been observed in eastern China but generally 2-4 years later than southern China, possibly due to the induction from there. More importantly, the H6N6 virus transmitted from waterfowl to land poultry is causing an endemic disease in poultry (H. Wu et al., 2016; Zhao et al., 2011; Zou et al., 2016).

The specific recognition and binding of hemagglutinin (HA) to sialic acid (SA) receptors on the host cells play a crucial role in determining the host range of influenza virus. Most AIV binds to avian-like receptors $\mathrm{SA} \alpha-2,3$ galactose(Gal), while the human influenza virus recognizes human-like receptors $\mathrm{SA} \alpha-2,6 \mathrm{Gal}$. For an effective human to human transmission, AIV must first acquire the ability to bind to the human-like receptor. Zou et al (Zou et al., 2016) found that 4 out of the 14 H6N6 AIV strains acquired the ability to recognize and bind to the human-like receptor. Wang et al (G. Wang et al., 2014) isolated 70 H6N6 viruses from the live poultry market in southern China from 2008 to 2011 and found that 48 strains (68.6\%) preferential bind to a human-like receptor. Bi et al (Bi et al., 2020) found that some H6N2 and H6N6 strains isolated in live poultry markets during 2016-2019 in China preferential bind to a human-like receptor. The molecular epidemiological investigation found that reassortment between H6 subtype and non-H6 subtype 
(especially H5N6 virus prevalent in Southern China) may easily occur (Li et al., 2019). Regarding the cases of human infection by H5N6 virus that occurred in 2014, it was found that H5N6 virus was generated by reassortment of $\mathrm{H} 6 \mathrm{~N} 6, \mathrm{H} 7 \mathrm{~N} 9 / \mathrm{H} 9 \mathrm{~N} 2$, and $\mathrm{H} 5$ viruses (Bi et al., 2016), which indicated that $\mathrm{H} 6 \mathrm{~N} 6$ acted as the progenitor of H5N6, a novel avian influenza virus infecting human. Meanwhile, some H7N9 viruses infecting humans have changed their NP and NS gene fragments by reassorting with H6N6 and H5N6 virus genes (Jin et al., 2017), which indicated that AIV H6N6 is currently in the dynamic status of evolution and is prone to reassorting with other subtypes of viruses, thus increasing the genetic diversity of the viruses. In 2011, swine-originated H6N6 virus A/SW/GD/K6/2010 (GDK6) was isolated in Southern China (Zhang et al., 2011). Gene sequencing analysis indicated that the virus originated from domestic ducks, and HA belongs to Group II virus of H6 Eurasian lineages. Animal experiments showed that the virus has limited transmissibility between ferrets (Sun et al., 2017), suggesting that the H6N6 virus could pass the interspecific barriers and infect humans. The seroepidemiological investigation found that the serum of exposed people from turkey farms in the United States was positive for H6 (Kayali G, 2010), and Li et al detected H6 antibody in the serum of 15,689 exposed people, resulting in a positive rate of $0.4 \%$ (Xin et al., 2015). Therefore, the possibility for humans to get infected by the H6N6 virus is increasing, while the risk for virus epidemic also rises.

H6N6 subtype AIV is widely prevalent in wild waterfowl and poultry in Eurasia, and its host range had gradually expanded to mammals, such as swine. Some viruses have even acquired the ability to recognize and bind to human-like receptor SA $\alpha-2,6$ Gal (G. Wang et al., 2014; Zhang et al., 2011; Zou et al., 2016). Following the continuous evolution of the virus, it remains unclear how readily it can develop the ability to cross interspecific barriers, thus affecting humans. In this study, the H6N6 subtype of avian influenza viruses isolated from chickens were sequenced and phylogenetically analysed, and the receptor-binding preference of the virus was analyzed. Then, the mice in vivo and human lung tissue in vitr o were inoculated with viruses to observe replication in mice and human lung tissue, as well as the molecular characteristics of the virus that infects mice and replicates in humans. Furthermore, we evaluated the potential of H6N6 viruses to infect humans and the key molecules that support binding to human-like SA $\alpha-2,6 \mathrm{Gal}$ preference and replication in humans.

\section{MATERIAL AND METHODS}

\subsection{Viruses}

Three strains of H6N6 subtype AIV isolated from chickens were selected in this study, including A/CK/JX/20490/2014(JX20490), A/CK/ZZ/1923/2015(ZZ1923), and A/CK/ZZ/346/2014 (ZZ346). Avian influenza virus H9N2 A/DK/GX/767/2010 (GX767), which had been previously confirmed to bind to avianlike $\mathrm{SA} \alpha-2$, 3-receptor, was used as a control for receptor-binding analysis. The swine influenza virus H1N1 A/SW/GX/3843/2011 (GX3843), which was previously confirmed to be able to infect mice, was used as the positive control for the in vivomice infection study. The human virus H3N2 A/ST/602/2005 (ST602), which can effectively replicate in humans and bind to avian-like $\mathrm{SA} \alpha-2,6$-receptor, was used as the in vitro human lung infection positive control.

Three strains of the H6N6 virus and one strain of H9N2 virus were passaged in 10-day-old embryonated chicken eggs; swine influenza virus H1N1 and human virus H3N2 were cultured in MDCK cells. HA subtype was identified by the HI test, and NA subtype was identified by direct sequencing.

\subsection{Genetic, phylogenetic and structural analyses}

Viral RNA was extracted with the RNeasy kit (Qiagen, Valencia, CA) and was reverse transcribed. PCR reaction amplification was performed by using segment-specific primers, and the products were purified. The whole virus gene was sequenced by the Illumina Solexa system. The reference sequences were retrieved from GenBank, https://www.ncbi.nlm.nih.gov/genomes/FLU/Database/nph-select.cgi?go=database. The sequence dataset was aligned by Muscle program (PMID: 15318951) followed by the manual adjustment. The best-fit nucleotides substitution model was selected from the 286 model candidates based on Bayesian information criterion (BIC) by using ModelFinder (PMID: 28481363). The Maximum-Likelihoood method 
was used to construct the phylogenetic trees implanted in the IQ-TREE 2.1.2 (PMID: 25371430). The phylogeny topological structure was supported by 10,000 times Ultrafast Bootstrap (UFBoot) (PMID: 23418397), 10,000 times Shimodaira-Hasegawa approximate likelihood ratio test (SH-aLRT) (PMID: 20525638), and the approximate Bayesian-like test (aBayes) (PMID: 21540409). The receptor-binding site (RBS) structure H6N6 ZZ346 virus was simulated based on H6 HA structure (PDB accession code: 5BR0) with molecular replacement and manual refinement using PyMOL 2.4.2 (Schrödinger, LLC).

\subsection{Receptor-binding analysis by HA assay}

An $\alpha 2,3$-specific sialidase can effectively eliminate SA $\alpha-2,3$ Gal, but retain SA $\alpha-2,6$ Gal. With $\alpha 2,3-$ sialidase-untreated TRBCs and $\alpha 2,3$-sialidase-treated TRBCs, the receptor-binding preference of the virus could be detected by HA's change titer. HA assays using resialylated turkey red blood cells (TRBCs) were performed as described previously (Suptawiwat et al., 2008) with some minor modifications. Briefly, $1 \%$ TRBCs solution was divided into $\alpha 2,3$-sialidase treated group and untreated group. $\alpha 2,3$-sialidase treated group was incubated with $1 \mathrm{U} 2,3$-specific sialidase (Takara, Japan) at $37{ }^{\circ} \mathrm{C}$ for $12 \mathrm{~h}$, while the untreated group with phosphgate-buffered saline (PBS) as a mock control. The TRBCs were washed with PBS three times. After centrifugation, two groups of TRBCs were reconstituted to $0.6 \%$ concentration. Then receptorbinding preference of the virus was detected by HA assay. Complete elimination of the SA $\alpha-2,3$-receptor on sialidase-treated TRBCs was confirmed by receptor staining and flow cytometry. The human influenza A virus $\mathrm{A} / \mathrm{ST} / 602 / 05(\mathrm{H} 3 \mathrm{~N} 2)$ and avian influenza virus DK/GX/767/2010 (H9N2) were used as controls for the HA assay.

\subsection{Receptor-binding analysis using a solid-phase direct-binding assay.}

Viral receptor-binding specificity was determined using the solid-phase direct binding assay as described previously (Bi et al., 2020) with minor modifications. Briefly, 96-well microtiter plates were coated with biotinylated glycans $\alpha 2-3$-SA receptors (Neu5Ac $\alpha 2-3$ Gal $\beta 1-4 G l c N A c \beta-C 3-P A A-b i o t i n, 3$ 'SLN) and $\alpha 2-6$-SA receptors (Neu5Ac $\alpha 2-6$ Gal $\beta 1-4 G l c N A c \beta-C 3-P A A-b i o t i n, 6$ 'SLN) (GlycoNZ Corporation, MD, USA). Then the virus dilutions containing $64 \mathrm{HA}$ units was added and the plates were incubated at 4 for $12 \mathrm{~h}$. Virusreceptor binding reaction was detected with human antisera against influenza viruses HA and HRP-linked rabbit-anti-human antibody (Beyotime Biotechnology) The reaction was stopped with 100ul Stop Solution for TMB Substrate, and the absorbance was determined at $450 \mathrm{~nm}$. The cutoff value for the glycan binding assays was the background value of the well with $100 \mathrm{ng}$ of glycopolymer in the absence of added virus. The human influenza A virus A/ST/602/05(H3N2) and avian influenza virus DK/GX/767/2010 (H9N2) were used as controls for the solid-phase direct binding assay.

\subsection{Animal experiments}

In this study, 60 6-week-old BALB/c mice were obtained through Vital River Laboratories, Beijing, China. Nasal swabs of all animals were inoculated in 10-day-old embryonated chicken eggs and Madin-Darby-canine kidney (MDCK) cell to confirm that mice were free of influenza viruses; HI assays were used to confirm that animals were negative for serum influenza virus antibody. This study was approved by the Animal Ethics Committee of Guangxi Medical University. Animal experiments were conducted in biosafety level $2^{+}$ containment facilities in strict compliance with the institute's guidelines for the care and use of laboratory animals.

To investigate the replication and infectivity of $\mathrm{H} 6 \mathrm{~N} 6 \mathrm{AIV}$ in mice, $60 \mathrm{BALB} / \mathrm{c}$ mice were divided into 5 groups $(n=12)$ by random number generator. Each animal was inoculated with one strain. The mock-infected group was inoculated with PBS. After anesthesia, each animal was intranasally inoculated with one strain at a dose of $10^{6} \mathrm{EID}$ in $0.2 \mathrm{~mL}$ of PBS or with $0.2 \mathrm{~mL}$ of PBS. The survial rate, weight change, temperature, and disease symptoms of animals were recorded daily during the 14-days course of the experiment. Animal nasal swabs were collected on days 1, 3, 5, and 7 post-inoculation and isolated and cultured in 10-dayold embryonated chicken eggs and MDCK. Three mice in each group were euthanized on days 3, 5, and 7 post-inoculation, respectively. Tracheal and lung tissues were collected and divided into two parts. One part was ground and centrifuged to obtain supernatant and then isolated and cultured in 10-day-old embryonated 
chicken eggs and MDCK. The other part was fixed in 10\% formalin solution at room temperature for 24 hours for pathological examination and virus protein detection. Sera were collected on day 14 post-inoculation, and $\mathrm{HI}$ tests were used to detect serum antibodies in the recovery period.

\subsection{Replication of $\mathrm{H} 6 \mathrm{~N} 6$ virus in human respiratory tissuein vitro}

This study was approved by the Medical Ethics Committee of Guangxi Medical University. Five lung tissue cases, which were collected during surgery, were provided by the First Affiliated Hospital of Guangxi Medical University. The selected specimens had no related respiratory tract infectious diseases. The specimens were sent to the laboratory immediately upon collection, and the suspected cancer tissues and/or other tissues with lesions were removed. The normal bronchial and lung tissues were cut into $0.2 \times 0.2 \times 0.2 \mathrm{~cm}^{3}$. Two blocks of bronchial and lung tissues from humans were selected to detect whether the samples were infected by influenza viruses. One of the blocks was grinded to isolate and culture the virus; the other one was used to detect viral antigen by immunohistochemistry and to ensure that the specimens used for the assay were free from influenza virus infection.

The bronchial and lung tissues were placed into a 6 -well cell culture plate, repeatedly rinsed with F-12K tissue culture medium containing antibiotics and L-glutamate, inoculated with $10^{6} \mathrm{TCID}_{50}$ of the virus in a volume of $500 \mu \mathrm{L}$ medium, and then cultured at $37^{\circ} \mathrm{C}$ and $5 \% \mathrm{CO}^{2}$ for $1 \mathrm{~h} .500 \mu \mathrm{L}$ of PBS was added into one well and used as a mock control. The tissue blocks were then rinsed with F-12K culture medium containing $0.2 \%$ TPCK-trypsin and 1\%BSA and further incubated with the above medium. Two tissue blocks were collected at $12 \mathrm{~h}, 24 \mathrm{~h}$, and $48 \mathrm{~h}$ post-inoculation of the virus, respectively, where one was ground in cold PBS and homogenated, and then the supernatant was collected and inoculated into 10-day-old embryonated chicken eggs and MDCK cells. TCID 50 was used to determine the virus titer. The other one was fixed in $10 \%$ formalin for 24 hours for pathological examination and detection of viral protein.

\subsection{Pathological examination and virus protein antigen detection}

Respiratory tract tissues of mice and lung tissues of humans were dehydrated, embedded, and serially sectioned with a thickness of $4 \mu \mathrm{m}$. The sections were stained with HE, and the pathological changes were observed under an optical microscope. An immunohistochemical method for the detection of viral protein was conducted as previously described (J. Wang et al., 2016). After antigen repairing, the primary nucleoprotein (NP) antibody (1:500) (kindly provided by the National Institute of Diagnostics and Vaccine Development in Infectious Diseases, Xiamen University) was added in sections. The sections were incubated overnight at $4^{\circ} \mathrm{C}$, followed by the addition of goat anti-mouse IgG-specific biotin conjugate (Calbiochem) (1:50), development by DAB stain, and counterstaining slightly by hematoxylin. Human lung tissue sections infected with influenza virus H5N1 were used as a positive control, and 10\% normal mouse serum was used as a MOCK control. The positive results were judged by the brownish coloring of the nucleus, and the cytoplasm around the nucleus appeared slightly brownish in color.

\section{RESULTS}

\subsection{Molecular feature and phylogenetic analysis of the virus}

Sequencing results showed that the HA cleavage site sequence of the three H6N6 strains was PQIETR/GL, with only a single basic amino acid belonging to the molecular characteristics of low-pathogenic AIV. No mutation occurred at 224,226, 228, 137, 138, and 190 sites (H3 numbering) of the main receptor binding sites. P186T, H156R, S263G mutation, and amino acid deletion at 158 of HA were found in the ZZ346 virus; these sites were not mutated in the other two viruses (Table 1 ). Whether mutations at these sites of HA would switch receptor specificity remains to be further studied.

The amino acid sequences of NA, PB2, and PB1-F2 of the three viruses were analyzed. The 11aa deletion in the stalk region of NA located at positions 59-69 was found in the ZZ346 virus strain; this deletion was not found in the other two viruses, JX20490 and ZZ1923. In this study, three strains of H6N6 viruses, including 627E, 271T, 701D of PB2-23 (Bussey, Bousse, Desmet, Kim, \& Takimoto, 2010; Steel, Lowen, Mubareka, \& Palese, 2009), 66N of PB1-F2 (Conenello, Zamarin, Perrone, Tumpey, \& Palese, 2007), 38I of PA (Tan et 
al., 2014) did not mutate but still showed the traits of replication and prevalence in poultry animals (Table 1 ).

The results of gene evolution analysis showed that three H6N6 subtypes of AIV were reassortment virus, and their gene segments were derived from group-II (ST2853-like) of Eurasian lineages (Figure1, 2 and Table2 ). For ZZ346 virus, the HA gene came from duck-originated H6N6 subtype in Fujian province in 2007, NA gene from H6N6 swine influenza virus in Guangdong province (A/Swine/Guangdong/K6/2010), while the other internal genes from clade of A/Duck/China/FJ2253/2014 (H6N6) in Fujian province. The HA gene of ZZ1923 came from clade of H6N2 subtype AIV in Sichuan province in 2014, the NA gene from the clade of duck-originated A/Duck/Guizhou/013/2014 (H6N6) in Guizhou province, and the PB2 gene from the A/Duck/Yamagata/061004/2014(H6N6) of Yamagata in Japan, and other internal genes from avian-originated (chicken, duck) and environment-originated H6N1, H6N6 subtype in Guangdong, Fujian, Guizhou, Guangxi, Hunan provinces in China. The HA gene of JX20490 came from clade of duck-originated A/Duck/Jiangxi/10304/2013 (H6N6) in Jiangxi province, the internal gene from clade of A/duck/Jiangxi/10304/2013(H6N6), except PB2 gene from clade of A/Chicken/China/GZ1063/2014 (H6N6).

\subsection{Receptor-binding specificity of $\mathrm{H} 6 \mathrm{~N} 6$ viruses}

Recently, a screening assay for receptor switching of avian influenza viruses was developed (Suptawiwat et al., 2008). The SA $\alpha-2,6$ or $\alpha$-2,3-binding preference could be distinguished by the change of HA titer in reaction with TRBCs and SA $\alpha-2,3 \mathrm{Gal}$-specific sialidase-treated TRBCs. Our results showed that H9N2 avian influenza virus GX767 and H3N2 human virus ST602 were preferentially bound to SA $\alpha-2,3 \mathrm{Gal}$ and SA $\alpha$ 2,6Gal receptors, respectively. Compared with HA titer of untreated TRBC of 1:128, HA titer of ZZ346 strain was 1:8 in SA $\alpha-2,3 \mathrm{Gal}$-specific sialidase-treated TRBCs, which only had $\alpha-2,6 \mathrm{Gal}$ receptors. Nevertheless, ZZ1923 and JX20490 strainses could not agglutinate SA $\alpha-2,3 \mathrm{Gal}$-specific sialidase-treated TRBCs (Figure 3 ). Our results indicated ZZ346 strain of the H6N6 virus could bind avian-like SA $\alpha-2,3 \mathrm{Gal}$ and human-like $\mathrm{SA} \alpha-2,6 \mathrm{Gal}$ receptors. We further confirmed $\mathrm{H} 6 \mathrm{~N} 6$ viruses preferentially bound to $\mathrm{SA} \alpha-2,3 \mathrm{Gal}$ or SA $\alpha-2,6 \mathrm{Gal}$ receptors by using a solid-phase binding assay. ZZ346 strain bound to both $\mathrm{SA} \alpha-2,3 \mathrm{Gal}$ or SA $\alpha-2,6 \mathrm{Gal}$ receptors, although their affinity for the $\mathrm{SA} \alpha-2,3 \mathrm{Gal}$ receptors was higher than that for the $\mathrm{SA} \alpha-2,6 \mathrm{Gal}$ receptors. But ZZ1923 and JX20490 strainses only bound to SA $\alpha-2,3 \mathrm{Gal}$ receptors (Figure 4 ). The results of the two assays were in agreement, confirming that ZZ346 strain of the H6N6 virus has acquired the ability to recognize the human-like receptors.

\subsection{BALB/c mice infected with H6N6 subtype AIV}

To investigate the replication of the $\mathrm{H} 6 \mathrm{~N} 6$ virus in mice, we inoculated groups of twelve 6 -week-old BALB/c mice with $10^{6}$ EID50 of each strain. Three mice in each group were sacrificed on day $3,5,7$ post-inoculation, and the virus was detected in the trachea and lung. The remaining three mice in each group were used to analyze weight changes, disease symptoms, and death after 14 days. After inoculation of H6N6 subtype AIV, BALB/c mice showed decreased activity, diet decline, coarse and disordered hair, but no disease symptoms weight loss and death. Their trachea and lung tissues were ground in homogenate and isolated in embryonated chicken eggs and MDCK, respectively. In embryonated chicken eggs, the virus was detected in the mice's tracheas and lungs inoculated with ZZ346; the HA titers on days 3, 5 post-inoculation were 1:32 and 1:16, respectively (JX20490 and ZZ1923 were not detected). Similarly, for MDCK isolation, the ZZ346 virus was detected in the lungs of mice; the HA titers on days 3, 5 post-inoculation were 1:16 and 1:8, respectively (JX20490 and ZZ1923 were not detected).

On day 14 post-inoculation, the serum of BALB/c mice was collected, and the HI test was used to detect the anti-influenza virus antibody in the serum. The results showed that although ZZ1923 and JX20490 strains were not be isolated, the serum of convalescent period was positive for the antibody. The antibody level induced by the ZZ346 strain was higher than that of JX20490 and ZZ1923 strains.

No obvious gross pathological changes were observed in the mice inoculated with JX20490 and ZZ1923, while a slight hyperemia focus of $1 \times 1.2 \mathrm{~cm}^{2}$ appeared in the left lung inoculated with ZZ346 on day 
5 post-inoculation. The trachea of mice inoculated with ZZ346 and JX20490 showed different degrees of tracheal mucosal congestion, edema, mucosal epithelial necrosis, and a small amount of inflammatory cell infiltration. However, no obvious pathological changes were found in the trachea of ZZ1923 inoculated mice (Figure 5 ). In mice inoculated with ZZ346, dilatation and congestion of small blood vessels, exudation of red blood cells, infiltration of inflammatory cells, widening of the alveolar wall, and interlobular septum in lung tissue were observed. Still, there were no obvious typical lesions in mice's lung tissue inoculated with JX20490 and ZZ1923 (Figure 5 ). Immunohistochemical detection of virus NP protein in tissue showed that the number of cells infected by ZZ346 was more than that infected by JX20490 and ZZ1923 (Table 3 and Figure 5 ). On days 3, 5, and 7 post-inoculation of ZZ346 strain, the NP protein of influenza virus were detected in the trachea, bronchus, and lung tissues of some mice. A small amount of virus NP protein could be detected in the trachea and bronchus of mice inoculated with JX20490, but no NP protein was detected in the lung. However, NP protein was not detected in the trachea, bronchus, and lung tissues of mice inoculated with ZZ1923. These results indicated that H6N6 viruses, especially the ZZ346 strain, could replicate in the respiratory system of mice without prior adaptation.

\subsection{Replication of H6N6 subtype AIV in human lung tissue}

Lung and bronchus tissues of humans were collected at 12h, 24h, and 48h post-inoculation of H6N6 viruses, after which they were ground. Their supernatant was then inoculated in 10-day-old embryonated chicken eggs and MDCK cells. In the isolation and culture of embryonated chicken eggs, the virus was detected in the lungs of humans inoculated with ZZ346, the HA titers on days 3 post-inoculation were 1:8; it was not observed for ZZ1923 and JX20490 virus. For MDCK isolation, three strains were not detected. The lung tissue inoculated with ZZ346 strain showed that lung tissue structure was destroyed, inflammatory cells such as lymphocytes and monocytes infiltrated in lung interstitial and bronchiolar mucosal epithelium necrosed and fell off. However, the obvious pathological changes of lung tissue inoculated with ZZ1923 and JX20490 virus strain were not observed. But there were no obvious histopathological changes in bronchial tissue inoculated with three strains of the H6N6 virus.

The immunohistochemical method was used to detect NP protein of influenza virus in bronchial and lung inoculated with three strains of the H6N6 virus. The virus NP protein was detected in alveolar cells of lung inoculated with ZZ346 virus strain, while was not detected in human lung tissues of JX20490 and ZZ1923 virus strain (Figure 6 ). NP protein of influenza virus was not detected in bronchus and bronchioles inoculated with three strains of H6N6. Our study suggested that the ZZ346 strain of the H6N6 virus, with binding to avian-like $\mathrm{SA} \alpha-2,3 \mathrm{Gal}$ and human-like $\mathrm{SA} \alpha-2,6 \mathrm{Gal}$, was effectively replicated in the human lung without prior adaptation.

\section{DISCUSSION}

H6N6 subtype AIV is widely prevalent in poultry, and its host range has expanded to mammals, such as swine. Undoubtedly, it has become an endemic disease of domestic fowl and domestic animals. Here, three chicken-originated H6N6 subtypes of AIV were multiple reassortment virus, and their gene segments were derived from group-II (ST2853-like) of Eurasian lineages. Terrestrial bird may have an intermediate host role in the cross-species transmission of influenza virus from birds to humans (Lam et al., 2013; Malik Peiris, 2009; Yang et al., 2017). At the same time, the molecular epidemiological investigation showed that H6N6 subtype AIV has been prevalent in terrestrial bird chickens (H. Wu et al., 2016). Therefore, the H6H6 virus from chickens may acquire the potential to infect humans. The switch of receptor-binding preference from avian-like SA2,3-Gal to human-like SA-2,6Gal is an key factor in avian influenza virus crossing interspecies barriers and efficiently transmitting to humans. The receptor-binding domains in the head of HA of influenza virus can specifically recognize and bind to avian-like SA2, 3-Gal and/or human-like SA2, 6-Gal receptor; yet, the molecular mechanism of receptor-binding preference switch in different avian influenza virus subtypes needs to be further elucidated. HA of H5N1 virus with N224K/Q226L mutations had a key role in switching receptor-binding preference from avian-like SA2,3-Gal to human-like SA-2,6Gal (Imai et al., 2012). HA with Q226L and G186V mutations in the H7N9 virus could result in virus binding to the human-like receptor (Dortmans et al., 2013), and HA with S137N, E190V, and G228S mutations of the H6N1 virus is essential 
in the process of acquiring the ability to recognize human-like virus receptors (Ni, Kondrashkina, \& Wang, 2015; F. Wang et al., 2015).

In this study, three strains of H6N6 virus, 224, 226, 228, 137 138, and 190 of HA receptor binding domains, have no mutation. However, P186T, H156R, S263G mutations, an amino acid deletion at 158 of HA were found in the ZZ346 strain. Interestingly, receptor-binding analysis by HA assay indicated that the ZZ346 strain of the H6N6 virus could bind to avian-like SA $\alpha-2,3 \mathrm{Gal}$ and human-like SA $\alpha-2,6 \mathrm{Gal}$ receptors. Some studies reported that HA with G186V mutations in H7N9 was able to bind to the human-like receptor (Dortmans et al., 2013). A combination of HA (H156N, S263R) and PA (I38 M) mutations might enhance the virulence of the virus in mice (Tan et al., 2014), suggesting that substitution of 186, 156, 263 of HA might be related to binding to human-like $\mathrm{SA} \alpha-2,6 \mathrm{Gal}$ receptors and effectively replicate in mammals. Therefore, we speculate that the chicken-originated ZZ346 virus strain of H6N6 virus with P186T, H156R, S263G mutations of HA could bind to avian-like SA $\alpha-2,3 \mathrm{Gal}$ and human-like SA $\alpha-2,6 \mathrm{Gal}$ receptors. Mutation or deletion of NA was observed during the adaptation of viruses to a new host, thus suggesting it crosses the host restriction (Hughes, McGregor, Suzuki, Suzuki, \& Kawaoka, 2001). Recently, amino acid deletion in NA's stalk region in some H6N6 subtype AIV was found ( $\mathrm{Li}$ et al., 2019). In this study, the 11aa deletion in the stalk region of NA located at positions 59-69 was found in the ZZ346 virus strain but not in JX20490 and ZZ1923 strains. The functional balance between HA and NA is crucial to the survival of the virus. HA affects virus binding to host cell, and NA affects progeny virus particles releasing from the host cell. Only when the two cooperate to reach the best balance state can the virus effectively replicate in the host cell, causing host infection onset (Gen et al., 2013). T271K, E627K, D701N of PB2 can enhance the polymerase activity, which favors enhancing the pathogenicity and transmission of the H6N6 virus in mammals. However, no substitution of these sites of PB2 of H6N6 virus was observed in this study, which was consistent with published papers (G. Wang et al., 2014; Zhang et al., 2011).

It remains unclear whether the ZZ346 strain of the H6N6 virus that can bind to avian-like SA $\alpha-2,3 \mathrm{Gal}$ and human-like $\mathrm{SA} \alpha-2,6 \mathrm{Gal}$ receptors could replicate and infect in mammals and humans. Accordingly, we selected the three chicken-originated $\mathrm{H} 6 \mathrm{~N} 6$ strains to inoculate BALB/c mice and human lung tissues. In the mice infection experiment, some strains of H6N6 influenza viruses were able to infect mice. After inoculating mice with ZZ346 strain, the virus and viral antigen NP were detected in the trachea and lung. In addition, antibodies in serum were detected on day 14 post-inoculation, indicating that the virus could effectively replicate in mice, thus causing infection. Regarding the JX20490 strain, NP protein was detected in the trachea and bronchial epithelial cells but not in the lungs. In addition, no virus was detected in the tracheal, bronchus, and alveolar tissue. The results showed that chicken-originated ZZ346 strain of H6N6 could directly infect mice without prior adaptation, which is consistent with a previous study $(\mathrm{H}$. $\mathrm{Wu}$ et al., 2016). Next, human lung tissue was inoculated with three strains of chicken-originated H6N6 subtype AIV in vitro . Lung tissue inoculated with ZZ346 strain showed local cell necrosis; virus and viral antigen NP were detected in the tissue. In contrast, virus and NP protein were not detected in the lung tissue after inoculation with JX20490 and ZZ1923 strains. Therefore, these data suggested that the ZZ346 strain was effectively replicated in the human lung without prior adaptation. At the same time, it provided evidence that P186T, H156R, S263G mutation, and amino acid deletion at 158 of HA were conducted to the switch of the H6N6 virus from binding to avian-like SA $\alpha-2,3 \mathrm{Gal}$ receptor to binding human-like SA $\alpha-2,6 \mathrm{Gal}$ and avian-like SA $\alpha-2,3 \mathrm{Gal}$ receptor. Based on the serum H6 antibody of the exposed population(Kayali G, 2010; Xin et al., 2015), our results indicated that the H6N6 virus acquired the potential to infect humans. 11aa deletion (59-69) in the stalk region of NA was found in ZZ346 strain, which also occurred in H5N6 virusinfected with human beings(Jin et al., 2017), speculating that the deletion at positions 59-69 in the stalk region of NA may be related to the infection of $\mathrm{H} 6 \mathrm{~N} 6$ in mammals, especially in humans. In this study, ZZ346 strain with HA variation (P186T, H156R, S263G mutation, and amino acid deletion at 158) and the 11aa (59-69) deletion in the stalk region of NA directly infected mice and effectively replicated in human lung tissue without prior adaption.

The first case of human infection with H6N1 avian influenza was reported on May 20, 2013, in Taiwan, China (Wei et al., 2013). The emergence of human infection cases with H6N1 indicated the unpredictability of in- 
fluenza virus transmission and novel viruses' potential threat. Some studies reported that the influenza virus causing pandemic was generated from avian-human (or-swine) influenza A virus reassortments (Smith et al., 2009), but the avian influenza virus involved in the reassortments is not necessarily a highly pathogenic avian influenza virus. Moreover, mild symptoms caused by low pathogenic virus infection can be easily ignored, increasing the chances of virus spread, adaptive mutation, and reassortment. Currently, the prevention and control of influenza pandemic are mainly focusing on the subtypes of bird flu viruses H5N1 and H7N9, which cause severe human diseases and deaths. However, due to unpredictability and the inadequacy of influenza virus-related knowledge, we cannot predict which subtype of influenza A virus will cause the next influenza pandemic. Although the $\mathrm{H} 6 \mathrm{~N} 6$ virus is a low pathogenic virus, it is widely prevalent in poultry. It repeatedly infects swine, which has the potential to evolve into a novel influenza virus infecting human beings. Therefore, this study confirmed that some chicken-originated H6N6 viruses might acquire the ability to recognize and bind to human-like receptors, thus increasing human infection risk. Our study emphasized the importance of continuous and intensive monitoring of the terrestrial bird H6N6 virus evolution to prevent transmission to humans.

\section{ACKNOWLEDGEMENTS}

This study has been supported by the National Natural Science Foundation of China (31660041), Guangxi Natural Science Foundation (2018GXNSFAA281300), Guangxi First-class Discipline Project for Basic medicine Sciences (No. GXFCDP-BMS-2018). The funders had no role in the study design, data collection and analysis, or preparation of the manuscript.

\section{CONFLICTS OF INTEREST}

The authors declare no potential conflicts of interest with respect to the research, authorship, and/or publication of this article.

\section{ETHICS STATEMENT}

The studies involving human participants were reviewed and approved by Medical Ethics Committee of Guangxi Medical University. The patients/participants provided their written informed consent to participate in this study.

\section{AUTHORS' CONTRIBUTIONS}

Lingxi Gao, Xijing Wang, Yigui Lin, Weijuan Zhong, Sugui Su, Yinchuan Zhu and Chengyi Li carried out the experiments, Lingxi Gao, Siyu Zhou, Xiaohui Fan and Zengfeng Zhang analyzed the data and wrote the manuscript. Zengfeng Zhang designed the study and revised the manuscript. All authors read and approved the final manuscript.

\section{DATA AVAILABILITY}

Genome sequences generated in this study are publicly available in the GenBank database under the accession numbers: MW494902-MW494909; MW494931-MW494938; MW495024-MW495031.

\section{REFERENCES}

Bahl, J., Vijaykrishna, D., Holmes, E. C., Smith, G. J., \& Guan, Y. (2009). Gene flow and competitive exclusion of avian influenza A virus in natural reservoir hosts. Virology, 390 (2), 289-297. doi:10.1016/j.virol.2009.05.002

Bi, Y., Chen, Q., Wang, Q., Chen, J., Jin, T., Wong, G., . . . Gao, G. F. (2016). Genesis, Evolution and Prevalence of H5N6 Avian Influenza Viruses in China. Cell Host Microbe, 20 (6), 810-821. doi:10.1016/j.chom.2016.10.022

Bussey, K. A., Bousse, T. L., Desmet, E. A., Kim, B., \& Takimoto, T. (2010). PB2 residue 271 plays a key role in enhanced polymerase activity of influenza A viruses in mammalian host cells. J Virol, 84 (9), 4395-4406. doi:10.1128/JVI.02642-09 
Chin, P. S., Hoffmann, E., Webby, R., Webster, R. G., Guan, Y., Peiris, M., \& Shortridge, K. F. (2002). Molecular evolution of H6 influenza viruses from poultry in Southeastern China: prevalence of H6N1 influenza viruses possessing seven A/Hong Kong/156/97 (H5N1)-like genes in poultry. J Virol, 76 (2), 507-516. doi:10.1128/jvi.76.2.507-516.2002

Claas, E. C., Osterhaus, A. D., van Beek, R., De Jong, J. C., Rimmelzwaan, G. F., Senne, D. A., . . . Webster, R. G. (1998). Human influenza A H5N1 virus related to a highly pathogenic avian influenza virus. Lancet, 351 (9101), 472-477. doi:10.1016/S0140-6736(97)11212-0

Conenello, G. M., Zamarin, D., Perrone, L. A., Tumpey, T., \& Palese, P. (2007). A single mutation in the PB1-F2 of H5N1 (HK/97) and 1918 influenza A viruses contributes to increased virulence. PLoS Pathog, 3 (10), 1414-1421. doi:10.1371/journal.ppat.0030141

Dortmans, J. C., Dekkers, J., Wickramasinghe, I. N., Verheije, M. H., Rottier, P. J., van Kuppeveld, F. J., ... de Haan, C. A. (2013). Adaptation of novel H7N9 influenza A virus to human receptors. Sci Rep, 3 , 3058. doi:10.1038/srep03058

Gao, R., Cao, B., Hu, Y., Feng, Z., Wang, D., Hu, W., . . . Shu, Y. (2013). Human infection with a novel avian-origin influenza A (H7N9) virus. N Engl J Med, 368 (20), 1888-1897. doi:10.1056/NEJMoa1304459

Gen, F., Yamada, S., Kato, K., Akashi, H., Kawaoka, Y., \& Horimoto, T. (2013). Attenuation of an influenza A virus due to alteration of its hemagglutinin-neuraminidase functional balance in mice. Arch Virol, 158 (5), 1003-1011. doi:10.1007/s00705-012-1577-3

Huang, K., Zhu, H., Fan, X., Wang, J., Cheung, C. L., Duan, L., . . . Guan, Y. (2012). Establishment and lineage replacement of $\mathrm{H} 6$ influenza viruses in domestic ducks in southern China. J Virol, 86 (11), 6075-6083. doi:10.1128/JVI.06389-11

Hughes, M. T., McGregor, M., Suzuki, T., Suzuki, Y., \& Kawaoka, Y. (2001). Adaptation of influenza A viruses to cells expressing low levels of sialic acid leads to loss of neuraminidase activity. J Virol, 75 (8), 3766-3770. doi:10.1128/JVI.75.8.3766-3770.2001

Imai, M., Watanabe, T., Hatta, M., Das, S. C., Ozawa, M., Shinya, K., . . . Kawaoka, Y. (2012). Experimental adaptation of an influenza H5 HA confers respiratory droplet transmission to a reassortant H5 HA/H1N1 virus in ferrets. Nature, 486 (7403), 420-428. doi:10.1038/nature10831

Jin, Y., Ren, H., Teng, Y., Hu, M., Peng, X., Yue, J., \& Liang, L. (2017). Novel reassortment of avian influenza $\mathrm{A}(\mathrm{H} 7 \mathrm{~N} 9)$ virus with subtype H6N6 and H5N6 viruses circulating in Guangdong Province, China. J Infect, 75 (2), 179-182. doi:10.1016/j.jinf.2017.05.006

Kayali G, O. E., Chorazy M, Gray GC. (2010). Evidence of Previous Avian Influenza Infection among US Turkey Workers. Zoonoses 83 Public Health, 57 (4), 265-272.

Lam, T. T., Wang, J., Shen, Y., Zhou, B., Duan, L., Cheung, C. L., . . . Guan, Y. (2013). The genesis and source of the H7N9 influenza viruses causing human infections in China. Nature, 502 (7470), 241-244. doi:10.1038/nature12515

Li, J., Quan, C., Xie, Y., Ke, C., Nie, Y., Chen, Q., . . . Bi, Y. (2019). Continued reassortment of avian H6 influenza viruses from Southern China, 2014-2016. Transbound Emerg Dis, 66 (1), 592-598. doi:10.1111/tbed.13037

Lupiani, B., \& Reddy, S. M. (2009). The history of avian influenza. Comp Immunol Microbiol Infect Dis, 32 (4), 311-323. doi:10.1016/j.cimid.2008.01.004

Malik Peiris, J. S. (2009). Avian influenza viruses in humans. Rev Sci Tech, 28 (1), 161-173. doi:10.20506/rst.28.1.1871

Ni, F., Kondrashkina, E., \& Wang, Q. (2015). Structural and Functional Studies of Influenza Virus A/H6 Hemagglutinin. PLoS One, 10 (7), e0134576. doi:10.1371/journal.pone.0134576 
Shen, Y. Y., Ke, C. W., Li, Q., Yuan, R. Y., Xiang, D., Jia, W. X., . . . Liao, M. (2016). Novel Reassortant Avian Influenza A(H5N6) Viruses in Humans, Guangdong, China, 2015. Emerg Infect Dis, 22 (8), 1507-1509. doi:10.3201/eid2208.160146

Smith, G. J., Vijaykrishna, D., Bahl, J., Lycett, S. J., Worobey, M., Pybus, O. G., . . . Rambaut, A. (2009). Origins and evolutionary genomics of the 2009 swine-origin H1N1 influenza A epidemic. Nature, 459 (7250), 1122-1125. doi:10.1038/nature08182

Steel, J., Lowen, A. C., Mubareka, S., \& Palese, P. (2009). Transmission of influenza virus in a mammalian host is increased by PB2 amino acids $627 \mathrm{~K}$ or $627 \mathrm{E} / 701 \mathrm{~N}$. PLoS Pathog, 5 (1), e1000252. doi:10.1371/journal.ppat.1000252

Sun, H., Kaplan, B. S., Guan, M., Zhang, G., Ye, J., Long, L. P., . . . Wan, X. F. (2017). Pathogenicity and transmission of a swine influenza A(H6N6) virus. Emerg Microbes Infect, 6 (4), e17. doi:10.1038/emi.2017.3

Suptawiwat, O., Kongchanagul, A., Chan-It, W., Thitithanyanont, A., Wiriyarat, W., Chaichuen, K., . . - Auewarakul, P. (2008). A simple screening assay for receptor switching of avian influenza viruses.J Clin Virol, 42 (2), 186-189. doi:10.1016/j.jcv.2008.01.014

Tan, L., Su, S., Smith, D. K., He, S., Zheng, Y., Shao, Z., . . . Zhang, G. (2014). A combination of $\mathrm{HA}$ and PA mutations enhances virulence in a mouse-adapted $\mathrm{H} 6 \mathrm{~N} 6$ influenza A virus. J Virol, 88 (24), 14116-14125. doi:10.1128/JVI.01736-14

Wallensten, A., Munster, V. J., Elmberg, J., Osterhaus, A. D., Fouchier, R. A., \& Olsen, B. (2005). Multiple gene segment reassortment between Eurasian and American lineages of influenza A virus (H6N2) in Guillemot (Uria aalge). Arch Virol, 150 (8), 1685-1692. doi:10.1007/s00705-005-0543-8

Wang, F., Qi, J., Bi, Y., Zhang, W., Wang, M., Zhang, B., . . . Shi, Y. J. E. J. (2015). Adaptation of avian influenza A (H6N1) virus from avian to human receptor-binding preference. 34 (12), 1661-1673.

Wang, G., Deng, G., Shi, J., Luo, W., Zhang, G., Zhang, Q., . . . Chen, H. (2014). H6 influenza viruses pose a potential threat to human health.J Virol, 88 (8), 3953-3964. doi:10.1128/JVI.03292-13

Wang, J., Wu, M., Hong, W., Fan, X., Chen, R., Zheng, Z., . . . Guan, Y. (2016). Infectivity and Transmissibility of Avian H9N2 Influenza Viruses in Pigs. J Virol, 90 (7), 3506-3514. doi:10.1128/JVI.0260515

Wei, S. H., Yang, J. R., Wu, H. S., Chang, M. C., Lin, J. S., Lin, C. Y., . . . Chang, F. Y. (2013). Human infection with avian influenza A H6N1 virus: an epidemiological analysis. Lancet Respir Med, 1 (10), 771-778. doi:10.1016/S2213-2600(13)70221-2

Wu, F., Zhao, S., Yu, B., Chen, Y. M., Wang, W., Song, Z. G., . . . Zhang, Y. Z. (2020). A new coronavirus associated with human respiratory disease in China. Nature, 579 (7798), 265-269. doi:10.1038/s41586-0202008-3

Wu, H., Lu, R., Peng, X., Peng, X., Cheng, L., Jin, C., . . . Wu, N. (2016). Isolation and genetic characterization of novel reassortant H6N6 subtype avian influenza viruses isolated from chickens in eastern China.Arch Virol, 161 (7), 1859-1872. doi:10.1007/s00705-016-2861-4

Xin, L., Bai, T., Zhou, J. F., Chen, Y. K., Li, X. D., Zhu, W. F., . . . Shu, Y. L. (2015). Seropositivity for Avian Influenza H6 Virus among Humans, China. Emerg Infect Dis, 21 (7), 1267-1269. doi:10.3201/eid2107.150135

Yang, L., Zhu, W., Li, X., Bo, H., Zhang, Y., Zou, S., . . . Shu, Y. (2017). Genesis and Dissemination of Highly Pathogenic H5N6 Avian Influenza Viruses. J Virol, 91 (5). doi:10.1128/JVI.02199-16

Zhang, G., Kong, W., Qi, W., Long, L. P., Cao, Z., Huang, L., . . . Wan, X. F. (2011). Identification of an H6N6 swine influenza virus in southern China. Infect Genet Evol, 11 (5), 1174-1177. doi:10.1016/j.meegid.2011.02.023 
Zhao, G., Lu, X., Gu, X., Zhao, K., Song, Q., Pan, J., . . . Liu, X. (2011). Molecular evolution of the H6 subtype influenza A viruses from poultry in eastern China from 2002 to 2010. Virol J, 8 , 470. doi:10.1186/1743-422X-8-470

Zou, S., Gao, R., Zhang, Y., Li, X., Chen, W., Bai, T., . . . Shu, Y. (2016). Molecular characterization of H6 subtype influenza viruses in southern China from 2009 to 2011. Emerg Microbes Infect, 5 (7), e73. doi:10.1038/emi.2016.71

zu Dohna, H., Li, J., Cardona, C. J., Miller, J., \& Carpenter, T. E. (2009). Invasions by Eurasian avian influenza virus H6 genes and replacement of the virus' North American clade. Emerg Infect Dis, 15 (7), 1040-1045. doi:10.3201/eid1507.090245

Table 1. Important amino acid sequences in HA, NA, PB2, PB1-F2 and PA of viruses

Table 2. Phylogenetic lineage of interal genes of ZZ346, ZZ1923 and JX20490

Table 3. Viral protein distribution in the respiratory tract of BALB/c mice inoculated with H6N6 influenza A viruses

The symbols,,-+++ , and +++ indicate that the numbers of cells with viral NP positive signal were 0,1 to 20,20 to 100 , and $¿ 100$, respectively, in each section.

Figure 1. The phylogenetic tree based on the HA gene of H6 influenza viruses. The viruses obtained in this study were highlighted in blue while the representative strains were highlighted inred. Each group was labeled to the right of the phylogeny. The length of scale bar represents the nucleotide substitutions per site. The values for the nodes are the consensus percentage support of SH-aLRT, aBayes, and UFBoot, respectively.

Figure 2. The predicted receptor-binding site (RBS) structure of H6N6 ZZ346 virus. The 130-loop, 190helix and 220-loop were highlighted in red. The important amino acids in the RBS pocket were labeled individually.

Figure 3. Characterization of the receptor-binding properties of influenza viruses was determinated by Hemagglutination test with $0.6 \%$ TRBCs treated and untreated with SA $\alpha-2,3 \mathrm{Gal}$-specific sialidase. The dashed line indicates the detection limit.

Figure 4. Receptor-binding specificity of H6N6 viruses using a solid-phase direct-binding assay. A: The human influenza A virus A/ST/602/05(H3N2) and avian influenza virus DK/GX/767/2010 (H9N2) were used as controls. B Receptor-binding properties of the representative AIV strains to human-like SA $\alpha 2-6$ and avian-like SA $\alpha 2-3$ receptors were tested using the solid-phase direct binding assay with trisaccharide receptors. Red and green lines represent human-like and avian-like receptors, respectively. Two replications presented similar results and the mean values were shown.

Figure 5. Pathological changes and virus replication in the trachea and lungs of mice inoculated with H6N6 viruses. Hematoxylin and eosin staining of the trachea (A1 to A3) and lungs (C1 to C3), immunohistochemical staining of the trachea (B1 to B3) and lungs (D1 to D3). The trachea and lungs of mices were inoculated with ZZ346 strain (A1 to D1), ZZ1923 strain (A2 to D2) and JX20490 strain (A3 and D3). The influenza NP antigen staining appeared as brown color (B1, B3 and D1). Scale bars, 50um.

Figure 6. H6N6 viruses replication in the lungs of human inoculated with $\mathrm{H} 6 \mathrm{~N} 6$ viruses in vitro. The immunohistochemical method was used to detect virus NP protein in lungs inoculated with ZZ346 strain (A), ZZ1923 strain (B2) JX20490 strain (C) and ST602 strain (D). The influenza NP antigen staining appeared as brown color (A and D). Scale bars, $50 \mu \mathrm{m}$. 

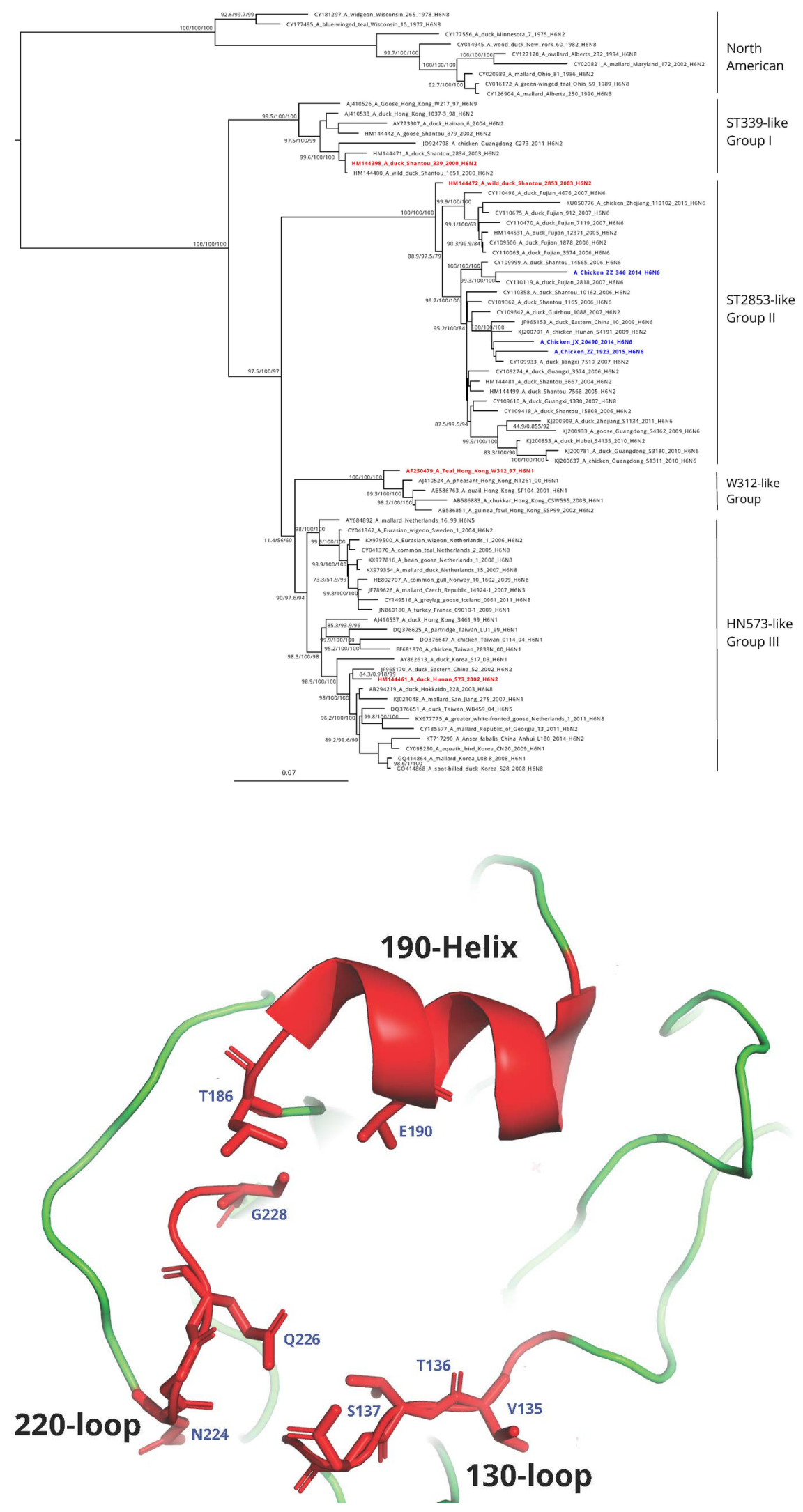

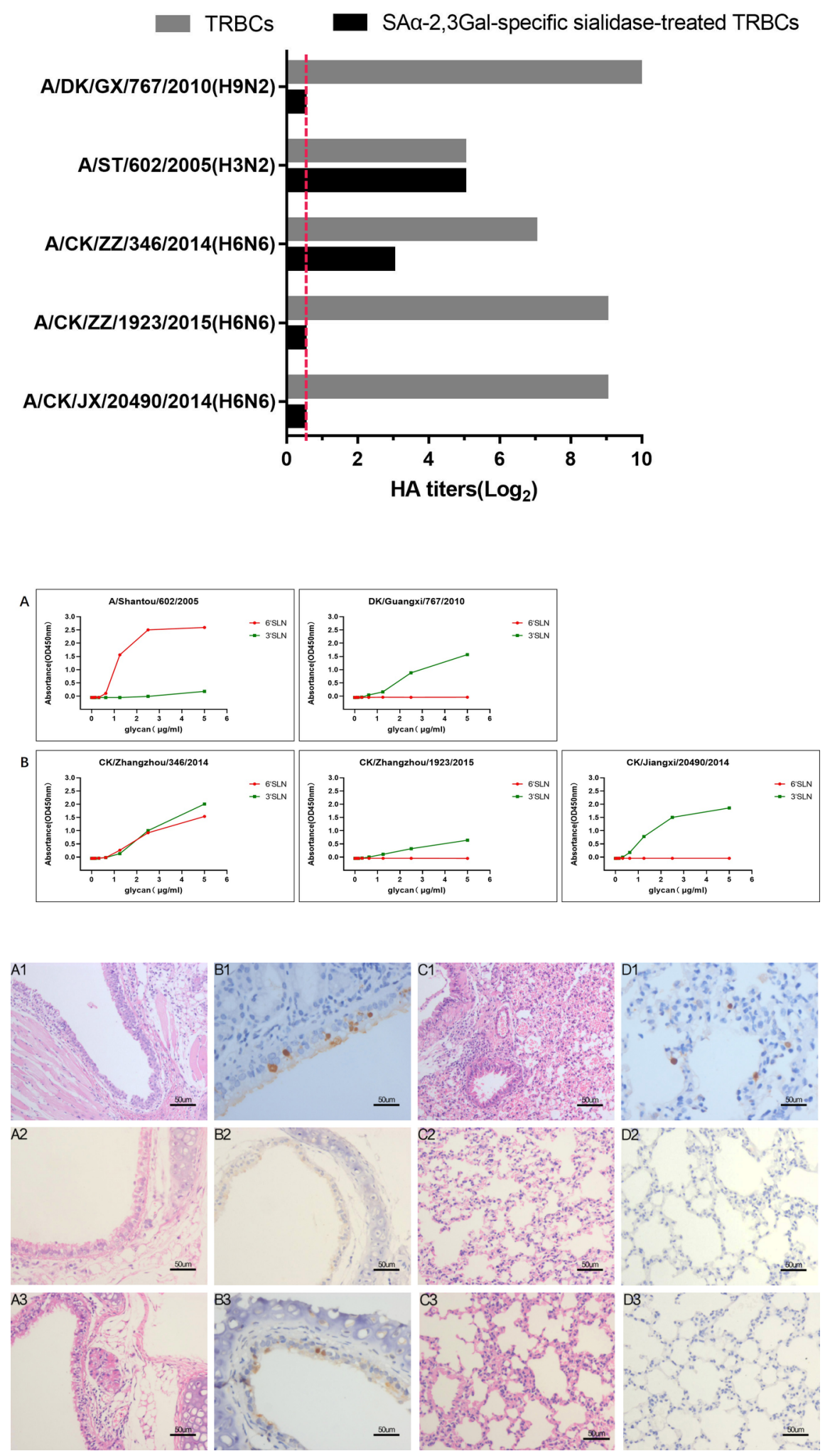

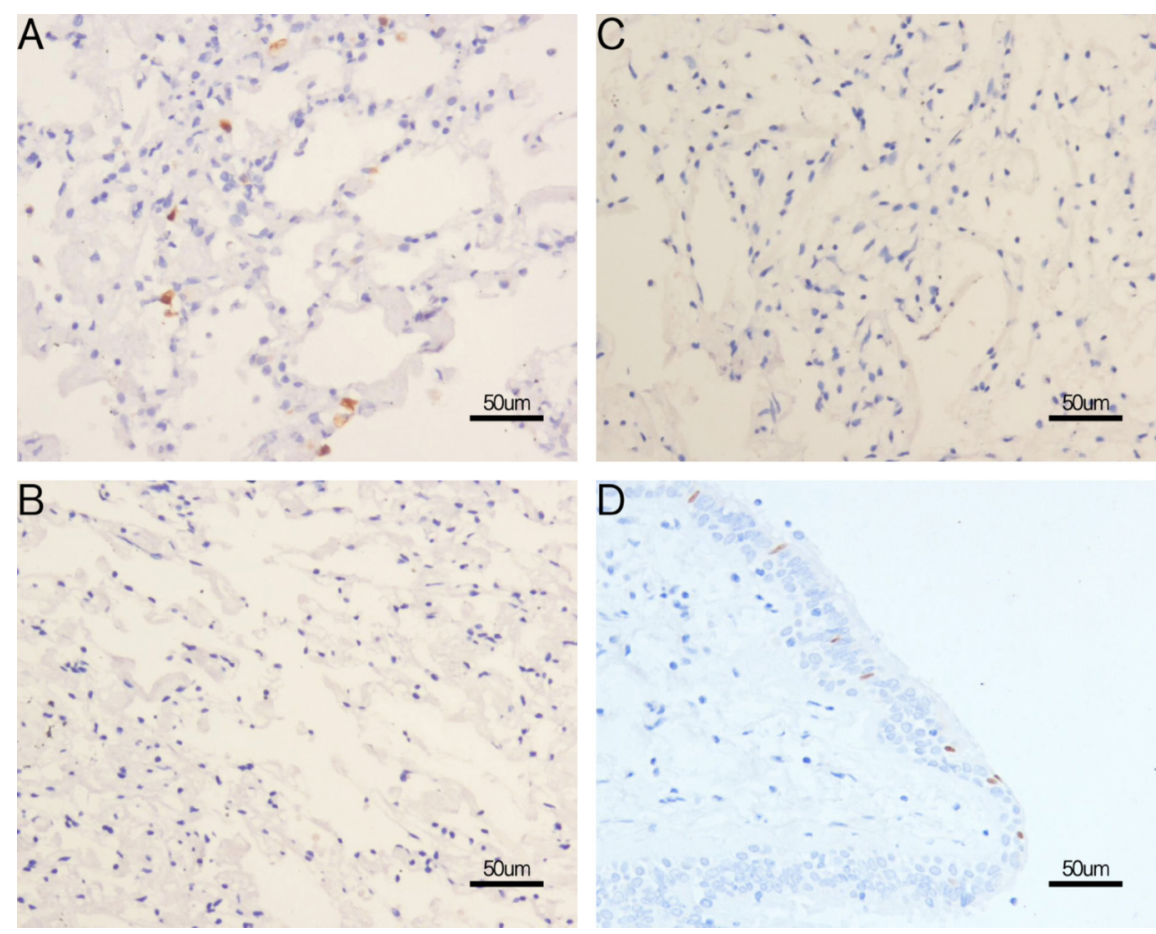

\section{Hosted file}

Table1-3.doc available at https://authorea.com/users/423597/articles/528927-influenzaa-h6n6-virus-isolated-from-chickens-replicate-in-mice-and-human-lungs-without-prioradaptation 